\title{
Alte und neue Guerilla in Lateinamerika - Folgen und Folgerungen aus der Revolution in Nicaragua
}

\author{
Von Peter Waldmann
}

Die Ursachen des Sturzes der Somoza-Diktatur sind inzwischen mehrfach analysiert worden, so daß es möglich erscheint, daran einige allgemeinere Uberlegungen hinsichtlich der Gründe des Scheiterns bzw. Erfolges lateinamerikanischer Guerillabewegungen zu knüpfen. Dies soll im Folgenden versucht werden. Der erste Abschnitt faßt die wichtigsten Voraussetzungen für den Zusammenbruch der Somoza-Herrschaft zusammen. Der breite Fächer der dabei zu erwähnenden Ursachen wird im zweiten Abschnitt durch die kritische Diskussion zweier gescheiterter Guerillafeldzüge (Venezuela, Argentinien) auf einige Schlüsselvariablen reduziert. Der dritte Abschnitt behandelt die Frage nach dem veränderten militärisch-politischen Stellenwert der Guerilla seit dem Sieg der Sandinisten (Juli 1979), wobei der Entwicklung in El Salvador und Guatemala besondere Aufmerksamkeit zugewendet wird. Die Argumentation geht nur auf jene Faktoren ein, die für die Entstehung und den Erfolg der Guerilla von Bedeutung sind; es wird also davon abgesehen, einen breiteren Úberblick über die Geschichte und Sozialstruktur sowie das politische System der beispielhaft herangezogenen Länder zu vermitteln. Diese im Interesse einer Straffung der Gedankenführung getroffene Entscheidung rechtfertigt sich daraus, daß es im deutschsprachigen Raum mittlerweile mehrere Handbücher und Lexika gibt, die dem Interessierten leicht erschließbares allgemeines Daten- und Informationsmaterial an die Hand geben (D. Nohlen, F. Nuscheler 1982; P. Waldmann 1982; zu Zentralamerika D. Boris, R. Rausch 1983).

\section{Erfolgsgründe der nicaraguanischen Revolution}

Einer auf H. Eckstein (1972: 18) zurückgehenden Einteilung folgend, unterscheiden wir strukturelle von akteurgebundenen Bedingungen des Revolutionserfolgs. Sie sind nicht immer sauber voneinander zu trennen, lassen sich aber andererseits auch nicht ohne weiteres ineinander auflösen. Eine dritte Kategorie nennen wir "operationale Bedingungen“. Darunter sind Umstände zu verstehen, die den Verlauf der Auseinandersetzung zwischen dem Regime und den Rebellen entscheidend prägten, ohne indes als Ursache im Sinne einer conditio sine qua non für den sandinistischen Sieg bezeichnet werden zu können. 


\section{Strukturelle Bedingungen}

Mehrere Autoren haben bei der Untersuchung der Ursachen der nic. Revolution zu Recht auf Besonderheiten in der Geschichte dieses Landes hingewiesen, das im 19. und frühen 20. Jahrhundert wegen seiner strategischen Schlüssellage innerhalb Zentralamerikas (z. B. im Hinblick auf den Bau eines zweiten Kanals) wiederholt Invasionen nordamerikanischer Truppen ausgesetzt war. Danach erklärt sich aus der Erfahrung langjähriger Besetzung des eigenen Territoriums, warum es im nic. Volk eine lebendige Tradition zähen und kompromißlosen Engagements gegen den nordamerikanischen Imperialismus und für die Wahrung der nationalen Unabhängigkeit gibt (D. PoloCheva, E. Süßdorf 1980: 21). Im Mittelpunkt dieser Tradition steht Augusto Cesar Sandino, jener berühmte Guerillaführer, der bei der letzten Intervention der USA (1927-1933) nicht ruhte, bis er die ausländischen Soldaten mit seinen Freischärlern außer Landes vertrieben hatte. Als in den 70er Jahren der bewaffnete Widerstand gegen das Somoza-Regime erstarkte, bot es sich aus mehreren Gründen an, als Symbolfigur Sandino zu wählen: Erstens wegen der Volksverbundenheit des Generals - die Mehrheit seiner Anhänger bestand aus Arbeitern und Bauern -, welche dem Anliegen der Guerilleros, die breite Bevölkerung zu mobilisieren, entgegenkam. Zweitens gab der Nationalismus und Antiimperialismus des Freiheitshelden ein ausgezeichnetes ideologisches Leitmotiv für den Kampf gegen das USA-hörige Somoza-Regime ab. Schon Mao Tse-tung (1974: 105, 137, 197) hatte in seinen kriegstheoretischen Schriften hervorgehoben, mit einem gegen einen äußeren Feind gerichteten Feldzug ließen sich ungleich größere Energien bei den Massen freisetzen, als wenn es sich nur um die Auseinandersetzung zwischen verschiedenen sozialen Klassen eines Landes handle. ${ }^{1}$ Drittens waren einige persönliche Eigenschaften Sandinos, vor allem seine Unbestechlichkeit und Lauterkeit, geeignet, um ihn gewissermaßen zum Gegenpol der durch und durch korrupten, eigennützigen Sippe der Somozas hochzustilisieren (ein Kontrastbild, das durch den Umstand verstärkt wurde, daß Sandino vom Begründer der Somoza-Dynastie, Anastasio S., auf heimtückische Weise ermordet wurde). Und schließlich erschien auch die von Sandino bis zur Perfektion entwickelte Kampftechnik gegenüber einem überlegenen Gegner, die Guerilla, zunächst als einzig erfolgversprechender Weg, um das festgefügte somozistische Herrschaftsgebäude zu erschüttern.

Ein zweiter den Niedergang des Regimes bewirkender Komplex von Faktoren läßt sich unter dem Stichwort Legitimitätsverfall zusammenfassen. ${ }^{2}$ Tatsächlich hatte die Diktatur von Anfang an Mühe, die Zustimmung der Bevölkerung zu finden. Dies lag zum

1 Daß die Mobilisierungskraft patriotischer Gefühle den Stimulierungseffekt von Klassenauseinandersetzungen weit übertrifft, läßt sich auch aus der Hartnäckigkeit ersehen, mit der kleine ethnische Gruppen wie die Basken oder Iren oft jahrzehntelang um ihre Eigenstaatlichkeit kämpfen.

2 Die Theoretiker der Protestgewalt haben meist klar gesehen, daß deren Erfolg mindestens so sehr von der Natur des angegriffenen Regimes wie von der Zahl, Energie und Opferbereitschaft der Rebellen abhängt. Siehe P. Waldmann 1977: 29. 
einen an ihrem Entstehungsmakel (ging sie doch auf einen Staatsstreich zurück) sowie an der Rücksichtslosigkeit, mit der die regierende Sippe ihre Machtposition behauptete, lag andererseits aber auch an der Unverfrorenheit, mit der sie politische Privilegien für privatwirtschaftliche Zwecke einsetzte, ein Sachverhalt, für den H. Jung (1980) den treffenden Ausdruck Bereicherungsdiktatur geprägt hat. Wenn die Familienherrschaft von der Mehrheit der Nicaraguaner dennoch einige Jahrzehnte lang geduldet wurde, so ist dies neben der weit ins 19. Jahrhundert zurückreichenden Tradition von Caudillismo und Willkürregierung vor allem mit der günstigen wirtschaftlichen Konjunktur während der 50er und 60er Jahre sowie damit zu erklären, daß sich die Somozas bei der Machtausübung zunächst eine gewisse Mäßigung auferlegten (T. W. Walker 1979: 316). $\mathrm{Da}$ sich das Legitimitätsdefizit ab 1970 zum Legitimationsverfall steigerte, hing mit der Reaktion des Regimes auf mehrere Herausforderungen zusammen. Die erste war das Erdbeben von Managua im Jahr 1972, das einen weltweiten Strom von Hilfsgeldern in das von der Katastrophe heimgesuchte Land auslöste. Regierung und Verwaltung waren nicht nur außerstande, diese Gelder sachgerecht zu verteilen und insbesondere den Bedürftigen zukommen zu lassen, sondern schlugen darüberhinaus aus der Notsituation Kapital, indem sie sich durch Unterschlagung von Spenden und die Ausnützung des bald darauf einsetzenden Baubooms aufs schamloseste bereicherten. Die Wahlfarce von 1974 sowie die Erklärung des Ausnahmezustandes (samt der Einschränkung mehrerer Grundrechte) als Antwort auf einen spektakulären Guerillaanschlag ließen das Regime weiter in die Isolierung geraten. Nach den schonungslosen, gegen breite Bevölkerungsgruppen gerichteten Unterdrückungskampagnen, mit denen die Nationalgarde sowohl auf Guerillaaktionen als auch auf Kritikvorstöße seitens der gemäßigten Opposition reagierte, entfiel schließlich jeglicher Schein einer institutionellen Bindung, entpuppte sich das Regime als reine Zwangsherrschaft.

Der Legitimitätsfirnis blätterte umso schneller ab, als die Regierenden sich ab Mitte der 70er Jahre außerstande zeigten, für weiteres Wirtschaftswachstum zu sorgen. Die Stagnation der Wirtschaft, die ab 1978 in eine Wirtschaftskrise mündete, gilt ebenfalls als einer der Gründe für den Zusammenbruch des Regimes (D. van Eeuwen 1980: 511 f.; H. Jung 1980: 142 f.) Die Unterschichten hatten ohnedies nur wenig von dem früheren wirtschaftlichen Aufschwung verspürt. Die Ausrichtung der (vorwiegend Zuckerrohr, Baumwolle und Kaffee produzierenden) Latifundien auf den Export hatte vielmehr zahlreiche Kleinbauern ihren Besitz gekostet und zu einer Verknappung der Grundnahrungsmittel, Reis und Bohnen, geführt. Mit der Rezession verschlechterte sich ihre Situation noch zusätzlich. Die Zahl der Arbeitslosen kletterte steil in die Höhe, während auch bei den Beschäftigten die steigende Inflationsrate nicht mehr durch Lohnerhöhungen aufgefangen werden konnte. Doch auch die Mittelschicht und das Großbürgertum waren von der Wirtschaftskrise betroffen, die teilweise unmittelbar mit dem Bürgerkrieg zusammenhing. Beispielsweise zerstörten Regierungstruppen bei der Niederschlagung des Aufstandes im Herbst 1978 durch Bombardierungen nicht weniger als 616 Geschäfte und setzten 30 Fabriken außer Betrieb (H. Jung 1980: 143). Die Folge der zunehmenden 
wirtschaftlichen Unsicherheit war eine intensive Kapitalflucht. Trotz wiederholter Kreditgewährung durch internationale Organisationen hatten die Auslandsschulden und das Zahlungsbilanzdefizit zu dem Zeitpunkt, als die Diktatur gestürzt wurde, ein solches Ausmaß erreicht, daß das Land praktisch bankrott war.

Als Strukturvariablen, die für den raschen Niedergang des Regimes von Bedeutung waren, sind schließlich auch die geringe Größe des Landes, seine geographische Lage sowie das internationale Klima in den entscheidenden Jahren der Offensive der Guerilla zu nennen. Alle drei Bedingungen hängen miteinander zusammen: Nur weil Nicaragua so klein und vergleichsweise rückständig war, hatte sich dort bis in die jüngste Zeit hinein ein Typus der Familiendiktatur halten können, den wir uns in den entwickelteren Flächenstaaten des Cono Sur (Argentinien, Uruguay, Chile) heute kaum mehr vorstellen können. Und nur weil diese in merkwürdiger Weise archaische Elemente und moderne Geschäftspraktiken vereinende Diktatur, allgemein sichtbar, das Wohlwollen der USA genoß, konnte die Kampfansage der Guerilla, die sich zugleich gegen den Hegemonieanspruch der nördlichen Supermacht richtete, jene Dimension einer Auseinandersetzung zwischen David und Goliath gewinnen, die den Sandinisten sowohl in Lateinamerika als auch in großen Teilen der europäischen Offentlichkeit so große Sympathien eintrug. Es darf nicht vergessen werden, daß die Zuspitzung des Konflikts in die Regierungszeit Carters fiel, dessen Menschenrechtspolitik erstmals mit dem traditionellen Prinzip nordamerikanischer Lateinamerikapolitik brach, die interamerikanischen Beziehungen ausschließlich als Instrument zur Wahrung eigener wirtschaftlicher und sicherheitspolitischer Interessen zu benutzen (W. Grabendorff 1971: 160 f.). Wie ein Autor bemerkte, fand seit dem Vietnamkrieg kein Befreiungskampf mehr eine so große internationale Resonanz und stieß auf eine solche Teilnahme, vor allem in der westlichen Welt, wie der Kampf des nic. Volkes gegen das Zwangsregime der Somozas (D. Castillo 1981: 14).

\section{Akteurbezogene Bedingungen}

Unter den Akteuren, deren Verhalten für den Sturz des Regimes entscheidend war, ist an erster Stelle der letzte regierende Chef des Somoza-Clans, Tachito Somoza, zu nennen. Ohne seine Irrtümer und falschen Reaktionen wäre es den Aufständischen weit schwerer gefallen, die Diktatur zu beseitigen. Nicht nur, daß er mit seiner maßlosen Bereichungsgier und durch die Mißachtung der bis dahin gültigen informellen politischen Spielregeln unnötigerweise eine Legitimitätskrise heraufbeschwor, er erwies sich, als diese eingetreten war, auch als unfähig, ihr wahres Ausmaß zu erkennen. Er unterschätzte die Bedeutung des institutionellen Scheins und überschätzte die Furcht der reformorientierten Opposition im Lande vor einer kommunistischen Revolution; er vertraute zu sehr auf den politischen Kontrolleffekt der Zwangsmittel in der Hand der Nationalgarde und täuschte sich hinsichtlich der Rebellionsbereitschaft des Volkes. Er ging von der uneingeschränkten Bereitschaft der USA zur Unterstützung seines Re- 
gimes aus, während ihn die Carter-Administration, wenngleich erst zu einem späten Zeitpunkt, zugunsten einer angestrebten bürgerlichen Alternativregierung fallen ließ. Kurzum, er war den Anforderungen der von ihm herbeigeführten Situation nicht gewachsen. Seine Kurzsichtigkeit geht deutlich aus dem blindwütigen Eifer hervor, mit dem er auf eine Eskalierung des Konfliktes setzte, auch nachdem sich die politisch-militärische Kräfterelation deutlich zu seinen Ungunsten verschoben hatte, so daß eine Polarisierung der Fronten seine Isolierung beschleunigen mußte.

Als Hauptgarant für die Stabilität der Diktatur galt die Nationalgarde (R. Millet 1977). Sie bildete aufgrund ihrer vorzüglichen Ausbildung und Bewaffnung zum einen, der unbedingten Ergebenheit gegenüber den Somozas (stets stand ein Mitglied der Familie an ihrer Spitze) zum anderen einen lebendigen Schutzschild zur Verteidigung der regierenden Sippe. Gleichwohl ist nicht zu übersehen, daß diese enge Verquickung von politischer und militärischer Macht die Exponiertheit des Regimes in dessen Niedergangsphase ungemein erhöhte. Kann sich die zivile politische Elite in anderen zentralamerikanischen Ländern darauf hinausreden, sie sei für die Gewaltexzesse der Streitkräfte nur bedingt verantwortlich, so mußte sich demgegenüber der Somoza-Clan die blinden und blutigen Vergeltungskampagnen, mit denen sich die Nationalgarde für erfolgreiche Guerillaaktionen an der Bevölkerung rächte, unmittelbar zurechnen lassen. Die stimulierende Wirkung, welche von dieser Möglichkeit der eindeutigen Identifizierung des Feindes auf die allgemeine Rebellionsbereitschaft ausging, sollte nicht unterschätzt werden. Die Rolle der USA, und speziell der Administration Carter in der Endphase der Auseinandersetzung muß ebenfalls differenziert gesehen werden. Einerseits besteht kein Zweifel daran, daß der Rückhalt, den das Regime als antikommunistische Ordnungsmacht Jahrzehnte lang in Washington fand, seine Festigkeit wesentlich erhöhte (W. M. Leo Grande 1979). Vor allem in den Augen der lokalen Ober- und Mittelschicht dürfte manche Schwäche und Blöße des herrschenden Clans dadurch ausgeglichen worden sein, daß er das Vertrauen der westlichen Führungsmacht genoß. ${ }^{3}$ Auf der anderen Seite war es just diese anbiedernde Haltung der Somozas gegenüber den Vereinigten Staaten, die dem gegen sie erhobenen Vorwurf, Handlanger des nordamerikanischen Imperialismus zu sein, Plausibilität verlieh und sowohl patriotisch gesinnte Jugendliche (vor allem Studenten) aus den Mittelschichten als auch die Unterschichten gegen das an den nationalen Interessen Verrat übende Regime mobilisieren half. Angesichts dieser ambivalenten Beurteilung der USA durch die nic. Bevölkerung wird man zögern, der unsteten, zwischen Stützen und Fallenlassen der Diktatur schwankenden Haltung Carters nach 1977 eine entscheidende Bedeutung für den Ausgang des Konfliktes beizumessen. Selbst wenn die USA auf die in Nicaragua sich stellenden Probleme nicht jeweils erst mit sechsmonatiger Verzögerung reagiert hätten (W. M. Leo

3 Die Somoza-Familie verbanden auch enge persönliche Beziehungen mit den USA. Der letzte Regierungschef des Clans, Tachito S., war beispielsweise in den USA ausgebildet worden und hatte gute Kontakte zu Kongreliabgeordneten; er war mit einer nordamerikanischen Frau verheiratet und stand mit mehreren nordamerikanischen Botschaftern auf freundschaftlichem Ful?. 
Grande 1977: 37), wenn es ihnen also gelungen wäre, durch ein schnelleres und energischeres Vorgehen gegen Tachito Somoza den gemäßigten Oppositionskräften zur Durchsetzung zu verhelfen, wäre nicht auszuschließen gewesen, daß der bürgerlichen in einem zweiten Schritt eine Revolution unter antiimperialistischem, sozialistischem Vorzeichen gefolgt wäre. ${ }^{4}$

Was die lateinamerikanischen Staaten betrifft, so wird man hingegen von einem überwiegend den Regimesturz begünstigenden Einfluß sprechen können, der allerdings erst in der "heißen" Phase der Auseinandersetzung zum Tragen kam, und zwar vor allem auf zwei Ebenen: einer militärisch-logistischen und einer politisch-symbolischen. In militärisch-logistischer Hinsicht erwies es sich von großem Vorteil für die Rebellen, daß ihnen die südlichen Nachbarländer, Costa Rica und das daran angrenzende Panama, als Rückzugs-, Aufmarsch- und Sammlungsgebiet zur Verfügung standen. Nicht von ungefähr konnte H. Bieber (1979: 20) die Feststellung treffen, das Engagement der Regierung Costa Ricas habe mehr zum sandinistischen Erfolg beigetragen als die Hilfe Kubas, von der wenig zu sehen gewesen sei. Auf der politischen Ebene profitierten die Aufständischen von dem neuen Drang der regionalen Mittelmächte, sich aus dem Hegemonieschatten der USA zu lösen und ein eigenes außenpolitisches Profil zu gewinnen (W. Grabendorff 1982: 251).

Mexiko und Venezuela sympathisierten offen mit ihnen, das letztgenannte Land lieferte sogar Waffen. Außerdem wirkte sich entscheidend zugunsten des internationalen Ansehens der Sandinisten aus, daß diese von Costa Rica und Mexiko diplomatisch anerkannt wurden.

Unter den innenpolitischen Akteuren, deren Verhalten das Regime destabilisierte, ist zunächst der mit dem Somoza-Clan nicht liierte Teil der Bourgeoisie zu nennen. Analysen des Machtrückgangs der Somozas weisen stets auf die Schlüsselbedeutung der Spaltung des bürgerlichen Lagers hin (statt aller D. Boris 1983: 282). Zugleich ist man sich darin einig, daß bis Anfang 1978, d. h. wenig mehr als ein Jahr vor dem Rücktritt T. Somozas, die Hauptinitiative und Mobilisierungskraft beim Widerstand gegen die Diktatur nicht den Guerilleros sondern der gemäßigten bürgerlichen Opposition zugekommen sei. Daß letztere sich mit zunehmender Heftigkeit gegen das Regime wendete, geht u. a. auf das bereits erwähnte Erdbeben von 1972 zurück. Hatte die herrschende Sippe bis zu diesem Zeitpunkt eine informelle wirtschaftliche Pfründenteilung akzeptiert, so dehnte sie nun ihr Wirtschaftsimperium auch in die bislang den anderen Gruppen des Bürgertums vorbehaltenen "Reservate" (beispielsweise den Bau- und den Banksektor) aus. Korruption und Unterschlagung staatlicher Gelder, eingefahrene Bereicherungspraktiken der Somozas und ihres Anhangs, erreichten ein Ausmaß, welches alle Regeln wirtschaftlicher Konkurrenz außer Kraft setzte und einer rationalen Planung und Kalkulation den Boden entzog (D. Polo Cheva, E. Süßdorf 1980: 27). Bedenkt man

4 Ob ein sozialistischer Revolutionsversuch gegen eine nichtsomozistische, bürgerliche, die volle Unterstützung der USA geniel.ende Regierung erfolgreich gewesen wäre, ist freilich eine Frage, die man durchaus unterschiedlich beantworten kann. 
weiter, daß sich T. Somoza ab 1974 immer ungehemmter über die verbliebenen institutionellen Schranken seiner Machtausübung hinwegsetzte, so liegt die Schlußfolgerung nahe, das langjährige Interessenbündnis zwischen dem Bürgertum und den Somozas sei deshalb zerbrochen, weil der letzte Regierungschef, T. Somoza, nicht mehr gewillt war, auf bestimmte Minimalforderungen bürgerlicher Gruppen hinsichtlich der Gewährleistung ihrer wirtschaftlichen und rechtlichen Sicherheit Rücksicht zu nehmen.

Der Positionswechsel eines Teils der Bourgeoisie von einer kooperativen Haltung bzw. systemloyalen Opposition zur offenen Infragestellung des Regimes ermunterte auch die abhängigen Mittelschichien (Angestellte, Beamte) sowie die Freiberuflichen, sich von diesem loszusagen. Hingegen waren es von solch generellen Trends unabhängige Motive, welche die Kirche, die lange Zeit gegenüber der Diktatur eine wohlwollende Neutralität gewahrt hatte, dazu bewogen, zunehmend kritischere Töne anzuschlagen. Der Klerus war über die Brutalität aufgebracht, mit der die Nationalgarde gegen die Bevölkerung im Anschluß an den erfolgreichen Guerilla-Coup vom Dezember 1974 vorging. Dem geharnischten Protest der Kirche gegen die ständige Verletzung der Menschenrechte kam umso größeres Gewicht zu, als er nicht allein vom niederen Klerus vorgetragen wurde. Auch die Bischöfe klagten das Regime wegen der Marterung und Tötung unzähliger Unschuldiger an und mahnten es zur Mäßigung.

In Nicaragua trat auch ein Akteur in Erscheinung, der, wenngleich er an sich als Protagonist revolutionärer Erhebungen gilt, bei Regimewechseln in Lateinamerika meist auf eine Statistenrolle verwiesen ist: das Volk. Wie H. Ortega (1980: 11), einer der sandinistischen Führer, berichtet, waren die Guerilleros selbst von der Energie und Spontaneität überrascht, mit denen die unorganisierte Bevölkerung, insbesondere die Jugendlichen aus den städtischen Unterschichten, gegen das Joch der Diktatur aufbegehrte. Ursprünglich hatten sie dem Volk eine Hilfsfunktion im Kampf gegen das Sippenregime zugedacht, während dessen Hauptlast auf ihren eigenen, den Schultern der bewaffneten Avantgarde liegen sollte. Als sie aber bemerkten, daß die Wellen des Volkszorns immer höher stiegen, definierten sie die eigene Aufgabe dahingehend um, diese verbreitete Wut nicht wirkungslos verpuffen zu lassen, sondern ihr eine bestimmte Richtung zu geben, sie zu kanalisieren und organisatorisch einzurahmen. Das erste Mal brach sich die Empörung der Massen nach der Ermordung des Führers der bürgerlichen Opposition, J. P. Chamorro, im Januar 1978 Bahn, als Tausende auf den Straßen von Managua zusammenströmten und den Rücktritt des Diktators forderten (T. W. Walker 1979: 325). Danach entlud sie sich noch mehrere Male, am heftigsten im Rahmen der im Mai 1979 beginnenden Schlußauseinandersetzung, die damit endete, daß die sandinistischen Kampftruppen in die bereits durch die Bevölkerung durch einen Aufruhr befreite Hauptstadt einziehen konnten.

Der Beitrag, den schließlich die im FSLN zusammengeschlossenen Guerilleros zum Sturz des Regimes leisteten, ist nicht leicht zu bestimmen. Kam ihnen beispielsweise nach V. Bye (1982) eine Schlüsselbedeutung zu, so würden wir ihre Rolle etwas vorsichtiger beurteilen. Wir sehen in ihnen nicht einen Akteur, der in der Lage gewesen wäre, 
den revolutionären Prozeß aus eigenen Kräften wesentlich voranzutreiben, sondern mehr den Katalysator, Koordinator oder, wenn man so will, die bewaffnete Vorhut der primär von anderen sozialen Gruppen getragenen politischen Umwälzung. Ihr Hauptverdienst bestand darin, daß sie durch ihre bewaffneten Aktionen die Erinnerung an den Freiheitskampf Sandinos wachhielten, bewiesen, daß die Diktatur verwundbar ist, und diese zu Gegenschlägen provozierten, von denen ein breiter Mobilisierungseffekt ausging. Im übrigen unterschied sich der FSLN, was seine soziale Zusammensetzung und Ziele angeht, kaum von anderen lateinamerikanischen Guerillabewegungen (Waldmann 1982: $103 \mathrm{ff}$.). Es handelte sich um eine Organisation mit einer sehr begrenzten Mitgliederzahl (bis Oktober 1978 rund 150, vorwiegend Studenten), die an die Stelle des Somoza-Regimes einen sozialistischen Staat setzen wollte (was inzwischen zumindest partiell geschehen ist). Bis 1977 war sie so wenig in breiteren Bevölkerungsschichten verankert, wie die meisten ihrer Vorgänger in anderen lateinamerikanischen Ländern. Anschließend zeigte sie jedoch eine bemerkenswerte Fähigkeit, die Umbruchstimmung erfassend, sich an die Spitze der revolutionären Bewegung zu setzen und dieser in wichtigen Augenblicken weitere Impulse zu geben. Mit anderen Worten: Die Hauptfunktion des FSLN lag im operativen Bereich.

\section{Operationale Bedingungen}

Da diesen Bedingungen in der Literatur oft besondere Aufmerksamkeit geschenkt wird (z. B. Bye 1982), können wir uns mit einer relativ knappen Aufzählung begnügen. Es ist wiederholt herausgestellt worden, daß die Schlagkraft der Guerillaverbände erheblich durch ihre Vereinigung und Unterstellung unter ein gemeinsames Oberkommando (im Frühjahr 1979) gesteigert wurde. Dieser Zusammenschluß war nur möglich, da jeder der drei Teilverbände ("tendencias") bereit war, Abstriche von seinem politischen und ideologischen Programm in Kauf zu nehmen. Die nämliche taktische Offenheit, ein Zug, der die Sandinisten vorteilhaft von den meisten früheren lateinamerikanischen Guerillaverbänden unterschied, ließ sie sowohl innerhalb Nicaraguas als auch international die Allianz mit gemäßigten, reformorientierten Kräften suchen. Auf der internationalen Ebene konnten sie die Unterstützung sozialdemokratischer Gruppen (z. B. der sozialdemokratischen Parteien Westeuropas und der Sozialistischen Internationale) gewinnen, während innenpolitisch ihr Ansehen dadurch aufgewertet wurde, daß ihnen von der "Gruppe der 12", einem bürgerlichen Honoratiorengremium, das 1977 mit einem antisomozistischen Manifest an die Offentlichkeit trat, politische Respektabilität bescheinigt wurde. Wie sie ihre ideologischen Grundüberzeugungen politischen Nahzielen unterzuordnen verstanden, so waren sie auch hinsichtlich des militärischen Vorgehens nicht auf ein bestimmtes Konzept festgelegt. Es wurde bereits darauf hingewiesen, daß sie ihre ursprüngliche Vorstellung, die Somoza-Herrschaft lasse sich nur durch einen militärischen Sieg nach klassischem Muster beseitigen, modifizierten, als sie der Rebellionsbereitschaft der Massen gewahr wurden. Die erst ab Ende 1977/Anfang 1978 von 
ihnen in Angriff genommene Aufgabe, die Bevölkerung für die bevorstehende Konfrontation zu formieren (sie in Nachbarschaftskomitees, Studenten-, Frauen-, Arbeiterverbänden zu organisieren, bzw. bereits bestehende Organisationen zu unterwandern und zu durchdringen), wurde so effizient gemeistert, daß Bye (192: $6 \mathrm{ff}$.) die nic. Revolution als das erste lateinamerikanische Beispiel einer gelungen Kombination von Guerilla und Massenmobilisierung bezeichnen konnte.

Etwas allgemeiner läßt sich der Vorzug der Vorgehensweise der Sandinisten dahingehend kennzeichnen, daß sie nicht blind auf die Eigendynamik der Gewalt setzten, sondern imstande waren, ihre Kampfaktionen in politische Zusammenhänge einzuordnen. Als beispielsweise das Regime 1977 Anstalten machte, gegenüber der bürgerlichen Opposition (u. a. durch die Aufhebung des Ausnahmezustandes) einzulenken, erkannten die Guerillaorganisationen blitzschnell die Gefahr der politischen Isolierung, die ihnen drohte, und begegneten ihr durch eine Intensivierung der Uberfälle und Anschläge (H. Ortega 1980: 8 f.). Durch dieses ständige Aufderhutsein, das unermüdliche Vorantreiben des Konfliktes, erzeugte der FSLN jenes kontinuierliche Wachstum der Protestund Aufruhrbewegung bis hin zur Massenerhebung in der Schlußphase der Auseinandersetzung, das von Mao Tse-tung (1974: 20, 54 f., 154 f.) als eine Schlüsselbedingung der Führung eines erfolgreichen Guerillafeldzuges bezeichnet wird. ${ }^{5}$

$\mathrm{Da}$ ein solch stetiges Wachstum erfolgen konnte, hing freilich auch mit zwei von den Sandinisten teilweise unabhängigen operationalen Vorteilen der Revolutionsbewegung zusammen. Der eine, die Existenz eines nahen Schon- und Rückzugsraumes (Costa Rica), wurde bereits erwähnt. Zumal in kleinen Ländern laufen Guerillaverbände, die sich vor gegnerischen Angriffen nicht über die Grenze eines mit ihnen sympathisierenden Landes in Sicherheit bringen können, Gefahr, von den Sicherheitskräften erdrückt zu werden. Ein zweiter wesentlicher Faktor war die Eindeutigkeit der von der Oppositions- und Widerstandsbewegung verfolgten Ziele: Die Entmachtung und Enteignung ${ }^{6}$ des Somoza-Clans, die Aufhebung des Belagerungszustandes und die Wiederherstellung der Grundrechte, die Auflösung der Nationalgarde und die Beseitigung der Korruption, waren so klare, in ihrer unmittelbaren Notwendigkeit einleuchtende Forderungen, daß es darüber auch zwischen ideologisch unterschiedlich orientierten Gruppen der Opposition schwerlich eine Auseinandersetzung geben konnte. In dieser Hinsicht finden Guerillabewegungen in anderen lateinamerikanischen Ländern, in denen die Machtverhältnisse weniger durchsichtig sind, weit weniger günstige Handlungsvoraussetzungen vor.

5 Die Bedeutung einer stetigen Expansion der Aufstandsbewegung für deren Erfolg ist sehr hoch einzuschätzen. Unter Umständen kann es für eine Guerillaorganisation ratsamer sein, lange Zeit Kräfte zu sammeln, um danach die Offensive kontinuierlich voranzutreiben, als nach einem schnellen Start anschließend in eine Stagnationsphase einzutreten.

$6 \mathrm{Da}$ es ein Umverteilungsobjekt in Form des immensen Besitzes der herrschenden Familie gab, hat das Zustandekommen einer Koalition zwischen dem FSLN und dem oppositionellen Bürgertum zweifellos erheblich erleichtert. Sozialistische Systemveränderungsversuche stoßen schon im Vorfeld auf weit größere Schwierigkeiten, falls sich die Umverteilungspläne der Revolutionäre nur auf Kosten des Besitzstandes der bürgerlichen Gruppen realisieren lassen. 


\section{Frühere Guerillabewegungen im Lichte der nic. Erfahrung}

Ist die nic. Revolution auf eine einmalige Konstellation von Bedingungen zurückzuführen oder lassen sich aus ihr allgemeinere Schlußfolgerungen ableiten? Diese Frage läßt sich nicht durch eine isolierte Betrachtung des nic. Falles beantworten, sondern macht einen Vergleich mit anderen, in ihrem Verlauf überschaubaren, d. h. im wesentlichen abgeschlossenen Guerillaepisoden erforderlich. Wir ziehen zwei Beispiele heran, bei denen (im Unterschied etwa zu dem Einnistungsversuch Che Guevaras in Bolivien) die Rebellen zumindest vorübergehend konkrete Erfolgschancen zu haben schienen: die Guerilla in Venezuela und Argentinien. Es versteht sich von selbst, daß es sich bei der Darstellung der Umsturzbewegungen in diesen beiden Ländern nur um grobe Skizzen ohne Anspruch auf die Berücksichtigung von Details handeln kann. Die Diskussion folgt der am Beispiel Nicaraguas entwickelten Einteilung in strukturelle, akteurgebundene und operationale Entfaltungs- und Erfolgsvoraussetzungen der Guerilla.

\section{Venezuela}

Die venezolanische Guerilla entstand zu Beginn der 60er Jahre. Ihren organisatorischen und personellen Rückhalt bildeten vor allem die Kommunistische Partei (PCV) und eine radikale Absplitterung (MIR) einer gemäßigten Linkspartei, der Demokratischen Aktion (AD). Zunächst rasch an Ausdehnung und militärisch-politischem Einfluß gewinnend, trat sie ab 1964 in eine Stagnationsphase ein, um 1969 praktisch von der Bildfläche zu verschwinden. ${ }^{7}$ Um die Ursachen ihres relativ raschen Niedergangs zu verstehen, muß kurz auf die politische Situation Venezuelas in den späten 50er und frühen 60er Jahren eingegangen werden. Das Land hatte nach dem Sturz des populistischen Diktators Perez Jimenez (Anfang 1958) eben zur Regierungsform der parlamentarischen Demokratie zurückgefunden. Während aber im Widerstand alle Parteien zusammengearbeitet hatten, schloß der Block der bürgerlichen Parteien die Kommunisten, als es um die Regierungsbildung ging, von einer Machtbeteiligung aus. Darauf propagierten die Kommunisten, die sich um die Früchte ihrer Untergrundarbeit geprellt fühlten, den allgemeinen Volksaufstand (Lindenberg 1968: 285). D. h. sie ermunterten ihre Anhänger dazu, der bürgerlichen in einem zweiten Schritt eine sozialistische Revolution folgen zu lassen, Parolen, mit denen sie bei Studenten und linken Gruppierungen anderer Parteien großen Anklang fanden. In der Tat bot die Regierung Betancourts, des Führers der AD, der 1958 das Präsidentenamt übernommen hatte, eine Reihe von Angriffsflächen: Sie machte sich, um den reaktionären Militärs keinen Putschvorwand zu liefern, nur zögernd an die Verwirklichung der zugesagten Reformvorhaben; sie hatte anfangs als

7 Zur Zeit ihrer maximalen Entfaltung soll sie 2000-3000 aktive Kämpfer umfaßt haben. K. Lindenberg 1968: 289. Die folgende Darstellung beruht vor allem auf dem Aufsatz von Lindenberg sowie den einschlägigen Kapiteln in den Werken von R. L. Lamberg 1971 und F. R. Allemann 1973. Siehe auch S. Ellner 1980. 
Hinterlassenschaft der verschwenderischen Diktatur mit wirtschaftlichen Schwierigkeiten zu kämpfen; sie büßte schließlich aufgrund ihres aggressiven Vorgehens gegen die Linke einen Teil ihrer ursprünglich breiten parlamentarischen Basis ein und mußte sich den Vorwurf der Hörigkeit gegenüber den USA gefallen lassen (F. R. Allemann 1974: 127). Gleichwohl war die Situation in keiner Weise mit jener vergleichbar, welche rund 20 Jahre später den Sieg der Sandinisten in Nicaragua ermöglichte. Denn die Regierung, gegen die sich die venezolanischen Linksextremisten wendeten, war nicht durch Gewalt, sondern aufgrund demokratischer Wahlen an die Macht gelangt. Hätte die revolutionäre Linke bereits gegen das Regime von Perez Jimenez gewaltsam aufbegehrt, so wäre ihre Ausgangslage vermutlich vorteilhafter gewesen. Der Caudillo war aber nicht in einer Volkserhebung verjagt, sondern vom Militär abgesetzt worden. Mochte die Mehrheit der Bevölkerung es auch als unfair empfinden, daß die Kommunisten, die in der Hauptstadt die zweitstärkste politische Kraft bildeten, beim politischen Machtpoker 1958 ausmanövriert worden waren, so ging ihre Empörung doch nicht so weit, daß sie sich eine andere politische Ordnung gewünscht hätte. Mit anderen Worten: Es fehlte an der Voraussetzung einer akuten Legitimitätskrise. Wie wenig die meisten Venezolaner daran dachten, das bestehende Regierungssystem infrage zu stellen, zeigte sich bei den Wahlen Ende des Jahres 1963, als trotz der mit Gewaltandrohungen verbundenen Aufforderung der Guerilla zum Wahlboykott mehr als $90 \%$ der Wahlberechtigten ihre Stimme abgaben.

Auch im übrigen waren die strukturellen Bedingungen für eine Volkserhebung nicht günstig. Ungeachtet des momentanen wirtschaftlichen Engpasses befand sich das Land in einer langfristigen wirtschaftlichen Aufwärtsbewegung, welche die Notwendigkeit eines prinzipiellen wirtschaftspolitischen Kurswechsels nicht als zwingend erscheinen ließ. Im Anschluß an die kubanische Revolution und unter dem Eindruck der von den USA eben verkündeten "Allianz für den Fortschritt", die als Immunisierungsstrategie gegen den kommunistischen Bazillus gedacht war, brachte die internationale westliche Offentlichkeit den Bemühungen der Guerilleros, Venezuela in einen zweiten sozialistischen Brückenkopf innerhalb Lateinamerikas zu verwandeln, wenig Sympathien entgegen. Die Abhängigkeit des Landes von den Vereinigten Staaten wurde im Unterschied zur Situation in Zentralamerika nicht als so drückend empfunden, daß von antiimperialistischen Parolen ein genereller Mobilisierungseffekt zu erwarten gewesen wäre. Schließlich hatte wegen des erreichten hohen Modernisierungsstandes sowie der fortgeschrittenen Zentralisierung und Urbanisierung Venezuelas ein Kleinkrieg auch in logistischer Hinsicht geringere Erfolgsaussichten als in agrarisch strukturierten Ländern. ${ }^{8}$ Was die sozio-politische Kräfteverteilung angeht, so konnten sich die Guerilleros zunächst durchaus gewisse Chancen ausrechnen, standen hinter ihnen doch zwei Parteior-

8 Womit nicht behauptet werden soll, dalh die für hochgradig urbanisierte und industrialisierte Staaten charakteristische Form des subversiven Kampfes, die Stadtguerilla, keine ernsthafte Herausforderung für die betreffende Regierung wäre. Mir ist aber kein Fall bekannt, in dem Stadtguerilleros das angestrebte Ziel, einen Regimesturz, erreicht hätten. 
ganisationen, darunter der über große organisatorische Erfahrung und einen beträchtlichen Personalbestand verfügende PCV. Die Kommunisten hatten u. a. Anhänger unter den Freiberuflichen und Intellektuellen, in den abhängigen Mittelschichten, den hauptstädtischen Elendsvierteln und vor allem bei den Studenten; ihr Rückhalt in den Gewerkschaften war ebenfalls nicht gering (F. R. Allemann 1974: 129). Die Bedeutung dieser Gruppen reichte jedoch bei weitem nicht aus, um die Situation "zum Kippen" zu bringen. Vor allem gelang es den Rebellen nicht, Teile des Bürgertums von der Notwendigkeit und Zweckmäßigkeit eines erneuten Regimewechsels zu überzeugen. Der "herrschende Block" spaltete sich nicht, Oberschicht und obere Mittelschicht identifizierten sich uneingeschränkt mit der frisch zurückgewonnenen parlamentarischen Demokratie. Daß von den Studenten, die sich der Guerilla anschlossen, nicht wenige aus alten angesehenen Familien stammten, läßt eher auf eine bestimmte Form der Bewältigung des Generationskonflikts als auf Entzweiungstendenzen innerhalb der gesellschaftlichen und politischen Führungsschichten schließen. Die Kirche, zu jener Zeit in Lateinamerika noch generell eine Stütze des Konservativismus, reagierte ebenfalls abwehrend gegenüber der "Kommunistischen Gefahr". Wie wenig die breite Bevölkerung für die Umsturzpläne der Guerilleros gewonnen werden konnte, geht, wie schon gesagt, aus den Wahlen von 1963 hervor. Die Deckung und Unterstützung, die städtische Terroristen anfangs bisweilen vonseiten der Bewohner der Elendsviertel erfahren hatten, wich, als darauf regelmäßige Razzien und Verhaftungen durch die Polizei erfolgten, einer indifferenten oder gar abweisenden Haltung. Auf dem Land fiel es den Guerillagruppen von vornherein schwer, Fuß zu fassen, teils weil die ländlichen Unterschichten keine materielle Not litten, teils weil sie bereits treue Anhänger der A. D. waren (K. Lindenberg 1968: 292; R. F. Lamberg 1971: 73 f.). Unter den außenpolitischen Machtfaktoren war es allein Kuba, das den Insurgenten Hilfe in jeder erdenklichen Form zukommen ließ (propagandistisch, durch Einsetzung von Ausbildern, in Form von Waffenlieferungen und Geldsummen). Dieser Beistand Kubas verstärkte aber eher noch die Isolierung der Guerilla gegenüber den übrigen Staaten in der Region, da die traditionellen Eliten Lateinamerikas, abgeschreckt durch Castros Wendung hin zum Sozialismus, zu Beginn der 60er Jahre jede Aufstands- und Umsturzbewegung mit äußerstem Mißtrauen verfolgten.

Die wenig erfolgversprechenden strukturellen Rahmenbedingungen und die ungünstige soziale Kräfterelation engten den Handlungsspielraum der Aufständischen beträchtlich ein, ohne daß ihnen dies zunächst bewußt war. Im übrigen liefert gerade die venezolanische Guerilla einen schönen Beweis dafür, daß viele der Züge, die als Manifestation des taktischen Geschicks der Sandinisten angeführt werden, zumindest ansatzweise schon bei früheren Guerillabewegungen zu beobachten waren. Dies gilt u. a. für die Anerkennung des Primats des Politischen gegenüber der blinden Gewaltanwendung (jedenfalls solange der PCV den Untergrundkampf leitete), für das Postulat einer einheitlichen Führung der revolutionären Kampfverbände (selbst wenn es in der Praxis nicht eingelöst wurde), das Nebeneinander von Stadt- und Landguerilla, Massenaktion und bewaff- 
netem Kampf, das gleichzeitige Operieren auf mehreren Ebenen (einer legalen politischen, einer symbolisch-kommunikativen, einer militärischen) sowie schließlich für die Taktik der Allianz mit unterschiedlichen, teilweise auch gemäßigten politischen Kräften (z. B. dem Militär). Allerdings waren all diese Elemente nicht in ein schlüssiges Gesamtkonzept eingefügt, sondern standen unverbunden nebeneinander oder wurden sukzessiv und gleichsam probeweise angewandt. Dieser Mangel an einer durchdachten Strategie spiegelte sich auch in der reichlich verschwommenen Zielsetzung der Guerilla wider, ein "revolutionäres, nationalistisches Volksregime" herbeiführen zu wollen, das den "Weg zum Sozialismus", der "unausweichlich" sei, zu öffnen habe (R. F. Lamberg 1971: 78). Angesichts einer so wenig klaren und zugkräftigen Definition der Nah- und Endzwecke, denen die Volkserhebung dienen sollte, erstaunt es nicht, daß die Bewegung der Rebellen nicht jene Schubkraft, jenen Sog zu entwickeln vermochte, die revolutionäre Prozesse auszeichnen. Statt kontinuierlich zu wachsen, schrumpfte sie, anstatt eine bedrohliche Polarisierung der gesellschaftlich-politischen Kräfte auszulösen, sank sie allmählich zu einem zweitrangigen Machtfaktor herab: Nach einem vielversprechenden Auftakt mit teilweise die ingeniösen Einfälle der Tupamaros vorwegnehmenden städtischen Guerillaaktionen, verlegten sich die Rebellen ab 1964 auf den Partisanenkampf in ländlichen Gebieten, wo sie zusehends in die Defensive und Isolierung getrieben wurden, um schließlich praktisch in Vergessenheit zu geraten.

\section{Argentinien}

Während die venezolanischen Guerilleros an keine historische Episode, keinen Namen aus der nationalen Vergangenheit anknüpften, ist die Entstehung der argentinischen Guerilla eng mit der Person Juan Domingo Peróns verbunden, jenes wegen seiner Politik sozialer Reformen und seiner nationalistischen Rhetorik bei den Massen beliebten Caudillo, der nach 10jähriger Amtszeit (1946-1955) von einer zivil-militärischen Allianz gestürzt wurde. Die Nachfolgeregierungen richteten sich nach der ungeschriebenen, von den Streitkräften diktierten Regel, daß die Peronisten auf jeden Fall von der Teilhabe an der politischen Macht auszuschließen seien. Trotzdem oder vielleicht gerade auch wegen dieses Verdikts hielten die argentinischen Unterschichten dem exilierten General unverbrüchlich die Treue. Im Laufe der Zeit entdeckten jugendliche Intellektuelle aus der Mittelschicht, deren Vorgänger einst zu den heftigsten Gegnern des Regimes gezählt hatten, ihr Interesse und ihre Sympathie für den Peronismus. Aus dieser Stimmungslage erklärt sich, daß 1969, inmitten einer Militärregierung, eine peronistische Guerillagruppe, die Montoneros, gegründet wurde, die sich die Rückführung des greisen Volksführers an die Macht und die Fortführung der 1955 abgebrochenen (vermeintlichen) nationalen und sozialen Revolution zum Ziel setzte. Gleichzeitig sprang eine Reihe weiterer Untergrundorganisationen, meist marxistischer bzw. trotzkistischer Coleur, aus dem Boden, die ebenfalls auf eine grundlegende politische und soziale Umwälzung 
hinarbeiteten. Die Untergrundbewegung dehnte sich rasch aus und erreichte eine Kampfstärke von mehreren tausend Aktiven. Sie trug mit ihren vorwiegend in Städten verübten Anschlägen und entsprechenden Propagandaparolen maßgeblich dazu bei, daß Wahlen ausgeschrieben wurden und, nach dem Sieg der Peronisten und einer Zwischenregierung, Perón selbst in das Land zurückkehren und die Regierungsgeschäfte übernehmen konnte. Als der General nicht den erwarteten sozialrevolutionären politischen Kurs einschlug, sondern statt dessen den Ausgleich mit der bürgerlichen Mitte suchte, zogen sich die enttäuschten Montoneros nach einer kurzen Waffenpause erneut in den Untergrund zurück, der von ihren trotzkistischen, mittlerweile im ERP versammelten Kampfgenossen nie verlassen worden war. Das verstärkte Eingreifen der Streitkräfte in den zunächst nur von Polizeikräften geführten Kampf gegen die Guerilleros drängte diese bald in die Defensive; nach dem militärischen Putsch vom März 1976 wurden sie in einer blutigen Säuberungskampagne schnell aufgerieben. ${ }^{9}$

Neben dem Einfluß des exilierten Caudillo, der seine jugendlichen Anhänger, solange er im Exil war, zu radikalem Vorgehen ermunterte und einer im einzelnen schwer präzisierbaren nationalen Bewußtseins- und Identitätskrise war für das Aufkommen und rasche Wachstum der Guerilla sicher der Umstand entscheidend, daß Argentinien seit 1966 von den Militärs regiert wurde. Diese Situation war insofern neu, als sich die Streitkräfte erstmals in der jüngeren argentinischen Geschichte nicht damit begnügten, als Drehscheibe zwischen den verschiedenen politischen Parteien und Gruppierungen zu fungieren oder die politischen Fäden hinter den Kulissen zu ziehen, sondern als Herrschaftsträger in eigener Sache auftraten (A. Rouquié 1978: 568 ff.). Dabei konnte man der Diktatur des Generals Onganía keine exzessive Härte vorwerfen. Sie hatte jedoch kurz nach der Machtübernahme die Universitäten unter Staatsverwaltung gestellt, wodurch linke Studenten in den Untergrund gedrängt worden waren; und sie irritierte die an das Recht der freien Meinungsäußerung gewöhnten Argentinier dadurch, daß sie nicht nur eine politische, sondern auch eine moralische Zensur ausübte. Bedenkt man weiter, daß es die nunmehr die Herrschaft ausübenden Streitkräfte waren, die durch ihr Veto der stärksten politischen Kraft des Landes, den Peronisten, seit über 10 Jahren den Zugang zur Regierungsgewalt versperrten, so kann man durchaus von einer durch den Putsch von 1966 heraufbeschworenen Legitimitätskrise sprechen. Zumindest ein Teil der mittelständischen Jugend betrachtete die Diktatur als ein Unrechtsregime, gegen das jede Art von Widerstand gerechtfertigt war.

Daß die Legitimitätskrise sich in Argentinien nach dem Einsetzen der Guerillaaktionen nicht weiter zuspitzte, ist nicht zuletzt der vorsichtigen Reaktion des Militärs zuzuschreiben, das, in sich zerstritten, verunsichert durch die wachsende Ablehnung seiner Herrschaft durch die breite Bevölkerung und erschreckt über die Gewalteruption, Anstalten traf, erneut zivilen Politikern das Feld zu räumen. Wurde der politische Rückzug der Waffenträger von den Guerilleros auch als ein Erfolg verbucht, der sie zu

9 Die Ausführungen über die argentinische Guerilla gehen auf eine eigene Untersuchung zurück. Siehe P. Waldmann 1978. 
einer Intensivierung ihrer Anschläge anstachelte (P. Waldmann 1978: 330 ff.), so stellten die Wahlen von 1973 und die bald darauf erfolgende Ubernahme der Regierung durch Perón das politische System doch wieder auf eine relativ solide Grundlage, die durch terroristische Provokationen allein nicht zu erschüttern war. Dies umso weniger, als andere strukturelle Rahmenbedingungen, vor allem die wirtschaftliche Lage sowie generell der Modernisierungs- und Entwicklungsstand des Landes, einer erfolgreichen Insurrektion eher im Wege standen. Die Situation war insoweit nicht unähnlich der für Venezuela beschriebenen: Argentiniens Wirtschaftswachstum blieb zwar hinter den von Politikern und Ókonomen angestrebten Wunschparametern zurück, die Lage war aber keineswegs desolat (T. Evers 1972: 66). Zudem hatten infolge der fortgeschrittenen Urbanisierung und Zentralisierung die bürokratischen Strukturen, vor allem die Streitund Sicherheitskräfte, eine Ausdehnung, Festigkeit und Durchsetzungskraft erlangt, die einen grundlegenden Systemwechsel nur unter der Voraussetzung einer breit angelegten Aufstandsbewegung von großer Dynamik möglich erscheinen ließen.

Für eine solche Erhebung fehlten jedoch die sozialen Vorbedingungen. Gewiß, die Montoneros, nach Mitgliederzahl und kämpferischem Potential der stärkere der beiden Guerillaverbände, wurden vom Linksperonismus getragen, ihnen eröffnete sich somit ein scheinbar unbegrenztes Feld zur Rekrutierung von Anhängern und Aktiven. Wie indes nachgewiesen wurde, bestand eine klare Scheidewand zwischen der Gewerkschaftsbewegung als der traditionellen organisatorischen Hauptstütze des Peronismus einerseits, und der erst ab 1970 sich formierenden peronistischen Jugendbewegung mit ihrem bewaffneten Arm, den Montoneros, andererseits (D. James 1976: 284). Die oft aus nationalistisch-katholischen Studentenzirkeln hervorgegangenen Guerilleros hatten nur begrenzten Zugang zur Arbeiterklasse. Wenn sie den Cordobazo von 1969, eine auf vielfältige Motive zurückgehende Erhebung von Industriearbeitern und Studenten in der wichtigsten Provinzhauptstadt des Landes (F. Delich 1974) als das Signal einer im Volk verbreiteten Insurrektionsbereitschaft auffaßten, so verkannten sie dabei den sozialkonservativen Grundzug in der Einstellung des argentinischen Arbeiters. Der größte Teil der Arbeiterschaft verehrte Perón als den politischen Führer, dank dessen Politik sozialer Reformen in den Jahren 1944-49 seine soziale Integration in das argentinische Gesellschaftsgefüge erfolgt war. Er erwartete von Peróns Rückkehr an die Macht eine Wiederauflage dieser Politik, d. h. eine Umverteilung des Volkseinkommens zu seinen Gunsten, nicht aber eine radikale Veränderung der bestehenden sozialen Ordnung. Deshalb reagierte er auf die Wiederaufnahme der terroristischen Aktionen durch die Montoneros ab 1974 teilweise mit Unverständnis. Ähnlich wenig Anklang fand die Fortsetzung des subversiven Kampfes bei den Mittelschichten. ${ }^{10}$ Ursprünglich, unter Onganía, hatten gewisse Sektoren dieser Schicht die Anschläge der jugendlichen Rebel-

Wenn hier von "den Mittelschichten" gesprochen wird, so bleibt dabei unberücksichtigt, daß dieses rund $40 \%$ der argentinischen Bevölkerung umfassende soziale Aggregat in zahlreiche Untergruppen zerfällt. Wie im Falle Venezuelas waren kleinere Gruppen der Freiberuflichen, Angestellten und Intellektuellen durchaus für die sozialrevolutionären Ziele der Guerillaverbände aufgeschlossen. 
len auf die Sicherheitskräfte zwar nicht ausdrücklich gebilligt, aber doch mit Schadenfreude verfolgt, wurde dem Militär auf diese Weise doch klar gemacht, daß seine Macht Grenzen hatte. Die Mittelschicht wie auch die traditionelle Oberschicht zog aus dem bewaffneten Aufbegehren der peronistischen Jugend vor allem den Schluß, der soziale und politische Frieden sei auf die Dauer nur gewährleistet, wenn die über den Peronismus verhängte Ächtung aufgehoben, dem greisen General eine echte politische Chance eingeräumt würde (wobei viele insgeheim auf eine Wahlniederlage der Peronisten hofften). Diese Revision ihrer früheren Haltung betrachteten sie bereits als ein großes Zugeständnis, allen weiterführenden Plänen der Linksperonisten, Peróns Regierungszeit zu einer sozialrevolutionären Umwälzung zu benützen, begegneten sie mit feindseliger Ablehnung. Sie applaudierten als erste, als Perón seine jugendlichen Anhänger öffentlich zurechtwies und zur Mäßigung und Disziplin ermahnte; und sie hatten keine Einwände dagegen, daß die Streitkräfte sich immer stärker in die Guerillabekämpfung einschalteten, um schließlich wieder die unmittelbare Herrschaft zu übernehmen. In kirchlichen Kreisen erfuhren die Guerilleros nur durch den sozial engagierten niederen Klerus Unterstützung, während sich die Bischöfe auf die Seite der Ordnungskräfte stellten (Waldmann 1978: 327). Da in den Nachbarländern Uruguay und Chile Anfang der 70er Jahre auf einen strikt antikommunistischen Kurs eingeschworene Militärjuntas die Macht übernommen hatten, standen die argentinischen Guerillaverbände, sowohl was den intranationalen als auch was den internationalen Kontext angeht, auf ziemlich verlorenem Posten.

Dabei wird man den Montoneros (weniger dem rigideren ERP) ein gewisses taktisches Geschick nicht absprechen können. Sie trieben eine offene Allianzpolitik gegenüber linken gewerkschaftlichen und parteipolitischen Gruppierungen, versuchten (allerdings erst zu einem relativ späten Zeitpunkt) bewaff nete Anschläge mit der Mobilisierung von Arbeitern und Slumviertelbewohnern zu verbinden, wählten ihre Opfer sorgfältig aus und schonten die breite Bevölkerung, blieben in ihren Zielsetzungen flexibel und versuchten dafür Verständnis in der internationalen Offentlichkeit zu wecken, errichteten ein ebenso umfangreiches wie gut ausgestattetes Netz von infrastrukturellen Einrichtungen und trieben die "Aktions-Repressionsschraube" nach bestem Vermögen in die Höhe. Ihre Hauptirrtümer bestanden darin, daß sie sich nicht rechtzeitig mit dem "historischen " Peronismus (d. h. den beiden ersten Amtszeiten Peróns) und den wahren Zielen des Caudillo auseinandersetzten (sonst hätten sie beizeiten ihre Fehlinterpretation seiner Absichten bemerkt) und daß sie die soziale Kräftelage, vor allem die Bereitschaft der Arbeiter, ihren Revolutionsaufrufen zu folgen, falsch einschätzten. Durch ihr ungedecktes aggressives Vorgehen lösten sie einen Gegenterror aus, auf dessen Konto mittlerweile weit mehr Opfer gehen, als sie selbst zu verantworten haben.

Versuchen wir, aus den zwei Fallskizzen in Verbindung mit der Analyse der nic. Revolution einige Schlußfolgerungen von allgemeiner Tragweite abzuleiten: Guerillaverbände können in Lateinamerika aus sehr unterschiedlichen Gründen und Konstellationen heraus entstehen: Unter Militärregimen oder zivilen Regierungen, in Phasen 
wirtschaftlicher Rezession oder wirtschaftlichen Aufschwungs, in abhängigen oder relativ autonomen Ländern, unter dem Einfluß eines international verbreiteten Protesttrends ebenso wie im Zeichen einer nationalstaatlich begrenzten Bewußtseins- und Identitätskrise. Zum Erfolg im Sinne eines politischen Machtgewinns führen ihre Gewaltaktionen aber nur unter ganz bestimmten Voraussetzungen:

1) Zum ersten scheint eine tiefe Erschütterung der Legitimitätsbasis eines Regimes, verbunden mit dem Aufbau einer entsprechenden Gegenlegitimation auf Seiten der Rebellen, unabdingbar zu sein. Diktaturen, die ja stets Legitimierungsschwierigkeiten haben, können diese Erschütterung hervorrufen, im allgemeinen reicht aber die bloße Tatsache der Außerkraftsetzung der Verfassung in den von autoritären Führungsmustern geprägten lateinamerikanischen Ländern nicht aus, um einen von so großer kollektiver Empörung getragenen Aufstand zu provozieren, daß nicht nur die Beseitigung der Regierung sondern des gesamten Herrschaftssystems beabsichtigt wird. Vielmehr müssen zusätzlich erschwerende Umstände hinzutreten, zu denen vor allem ein Ubermaß an Korruption und Vetternwirtschaft, eine ungezügelte Repression sowie eine ineffiziente, das Land in eine Krise führende Wirtschaftspolitik zählen. Auf der anderen Seite ist nur dann mit einem bewaffneten Vorgehen breiter Schichten gegen ein Regime zu rechnen, wenn die Führer der Rebellen es verstanden haben, der Masse ein glaubwürdiges Gegenmodell zu präsentieren, d. h. in ihnen die zwingende Vorstellung von der Uberlegenheit und Realisierbarkeit der angestrebten wirtschaftlichen und politischen Machtordnung zu erzeugen.

2) Eine weitere, weit weniger präzis bestimmbare strukturelle Voraussetzung eines revolutionären Umschwungs sehen wir darin, daß es sich um ein relativ kleines, nicht allzu entwickeltes, wirtschaftlich offenkundig von überlegenen Industriestaaten abhängiges Land handeln muß. Es kann nicht nur ein Zufall sein, daß in den südamerikanischen Flächenstaaten auch Guerillabewegungen, die bereits eine beträchtliche Größenordnung erreicht hatten, rasch eliminiert wurden, ${ }^{11}$ während in Zentralamerika die aufständischen Kampfeinheiten, ob siegreich oder nicht, mehrfach zu einem gewichtigen militärisch-politischen Machtfaktor geworden sind. Dies dürfte sich u. a. daraus erklären, daß in hochindustrialisierten Staaten als subversive Kampfmethode nur die Stadtguerilla infrage kommt, die in keinem der bisher bekannten Fälle einen Regimesturz bewirken konnte; daß moderne Flächenstaaten über einen hochgradig bürokratisierten Sicherheitsapparat (Militär, Polizei, Geheimdienste etc.) verfügen, für den eine Kaderguerilla keine ernsthafte Gefahr darstellt; und daß schließlich nur kleine Länder jene intensive wirtschaftliche und politische Außenabhängigkeit aufweisen, die erforderlich ist, um ein allgemeines Gefühl nationaler Schmach und Erniedrigung sowie entsprechende Reaktionen kollektiver Empörung zu erzeugen.

11 Auch die Tupamaros waren letztlich erfolglos und wurden ziemlich rasch vernichtet, obwohl Uruguay kein "ausgedehnter Flächenstaat" ist. Die eben genannten Erfolgsbedingungen für die Guerilla: klein, unterentwikkelt, abhängig dürfen jedoch nicht nur einzeln vorliegen, sondern müssen in cumulo gegeben sein. Uruguay ist zwar klein, aber von der Topographie her für eine Landguerilla ungeeignet und zudem relativ entwickelt. 
3) Auf der Ebene der Machtakteure ist es für einen Erfolg der Rebellen unerläßlich, daß es ihnen gelingt, einen Teil der gemäßigten, reformorientierten Kräfte in ihr Lager herüberzuziehen. Ohne eine Spaltung des bürgerlichen Blocks können sie allenfalls ein soziopolitisches Machtpatt, jedoch kein Ubergewicht erreichen. Von den unter 1) aufgeführten Ursachen des Legitimitätsschwundes eines Regimes sind die bürgerlichen Gruppen besonders reizbar durch die Einschränkung der Meinungsfreiheit, das Uberhandnehmen der Korruption sowie eine dilettantische bzw. für ihre Interessen schädliche Wirtschaftspolitik. Sie reagieren auch sensibler als andere Schichten auf Schwankungen des Ansehens einer Regierung in der internationalen öffentlichen Meinung. Dagegen akzeptieren sie um der Aufrechterhaltung der öffentlichen Ordnung willen zumindest zeitweise einen vergleichsweise hohen Pegel staatlicher Repression, solange ihre eigene Sicherheit nicht gefährdet erscheint. Nur ein kleiner Teil der gemäßigten Kräfte, vor allem die Kirche, Intellektuelle und Vertreter freier Berufe, messen die Legitimität eines Herrschaftssystems primär daran, wieweit es die Grund- und Menschenrechte respektiert.

4) Uber die Rolle des "Volkes" im Rahmen erfolgreicher Guerillafeldzüge lassen sich (da es bisher außer in Nicaragua nur ausnahmsweise, insbes. bei der mexikanischen und bolivianischen Revolution, in Erscheinung getreten ist) nur vorsichtige Mutmaßungen anstellen. Unter diesem Vorbehalt sei die These vertreten, daß ohne die Mobilisierung breiter Bevölkerungsschichten ein revolutionärer Umsturz kaum zu bewerkstelligen ist. Es bedarf dieser Mobilisierung desto mehr, je wehrfähiger und in bürokratischen Strukturen verankert das angegriffene Regime ist. ${ }^{12}$ Im allgemeinen sind die lateinamerikanischen Unterschichten weniger für radikale Änderungen aufgeschlossen, als Marxisten wahrhaben wollen. Sie können nur unter bestimmten Bedingungen zu kollektiven Aktionen getrieben werden, die über den Schutz ihrer unmittelbaren Interessen oder Gefolgschaftsbekundungen gegenüber einem charismatischen Führer hinausgehen. Solche Voraussetzungen sind u. a.: daß sie aufgrund extremer Unterdrückung und materieller Not keine andere Úberlebenschance sehen als den Anschluß an die Rebellen; daß sie noch nicht in eine bereits bestehende Partei oder sonstige Organisation integriert sind, die Status-quo-Interessen verteidigt; schließlich daß ihre Motivation durch an Emotionen appellierende Ziele geweckt wird, wozu sich nationalistische Parolen im allgemeinen besser eignen als sozialistische Versprechungen.

5) Die letztgenannte Bedingung leitet bereits zu den operationalen Voraussetzungen erfolgreicher Guerillabewegungen über, von denen nur zwei besonders wichtige heraus-

12 Bei der Formulierung dieser Aussage dachten wir vor allem an die Kubanische Revolution von 1958/59, die bekanntlich unter geringer Massenbeteiligung durchgeführt wurde. Daß\} sie dennoch erfolgreich war, erklärt sich aus der morschen Struktur des Batista-Regimes, das nur eines kleinen Stol.jes bedurfte, um in sich zusammenzubrechen. Da sich nach dem Erfolg der kubanischen und in jüngster Zeit der nicaraguanischen Guerilleros die meisten Regime militärisch nach besten Kräften gegen Aufstandsbewegungen gewappnet haben, dürfte es heute weit schwieriger, ohne die Unterstützung breiter Bevölkerungsschichten fast unmöglich sein, die bestehenden Herrschaftsstrukturen zu beseitigen. Die restlichen vier Bedingungskomplexe lagen im kubanischen Fall zumindest ansatzweise vor. Vgl. R. F. Lamberg 1971: 12 f.; B. Goldenberg u. K. El.3er 1969. 
gegriffen seien. Zum ersten ist dies die Definition eines einprägsamen, verständlichen, realisierbaren Zielkatalogs unter Verzicht auf komplizierte theoretische Ableitungen (ob dies möglich ist, hängt freilich nicht zuletzt von der Durchsichtigkeit der bestehenden Machtverhältnisse ab); zweitens bedarf die Guerilla zu ihrer Entfaltung inner- und/oder außerhalb des eigenen Territoriums ungefährdeter Rückzugs-, Schon- und Aufbauzonen, um nicht, solange sie den Sicherheitskräften unterlegen ist, erdrückt und eliminiert zu werden. Hingegen zählen wir die "Provokations-Repressionsspirale", auf die von den Guerilleros in Südamerika so große Hoffnung gesetzt wurde, nicht zu den operationalen Erfolgsprinzipien, handelt es sich dabei doch um ein zweischneidiges Schwert. Nur wo sich bereits ein Kräfteübergewicht der Opposition abzeichnet, kann durch eine forcierte Polarisierung der Fronten der Sog zugunsten der Aufständischen verstärkt und das Regime in die Isolierung getrieben werden. Ansonsten laufen die Rebellen durch eine blinde Steigerung der Gewaltaktionen Gefahr, selbst von ihrer potentiellen sozialen Basis abgeschnitten zu werden oder können bestenfalls eine politisch-militärische Pattsituation stabilisieren.

\section{Entwicklung der Guerilla in Zentralamerika seit der nic. Revolution}

Der Erfolg der Sandinisten hat zu einer erheblichen Verhärtung der Fronten zwischen den Insurgenten einerseits, den Verteidigern der gegenwärtigen Herrschaftsordnung andererseits in Mittelamerika geführt. Die ersteren sehen sich durch das Beispiel Nicaraguas nicht nur erneut in ihren Hoffnungen auf einen gewaltsamen Systemwandel bestätigt, sondern haben dazu einen konkreten Verbündeten in der Region gewonnen. Die Gegenseite bietet angesichts der gesteigerten Bedrohung ihr gesamtes Repressionspotential auf und sucht ebenfalls nach auswärtiger Unterstützung, um die Gefahr eines Machtwechsels zu bannen. Die wichtigsten Veränderungen gegenüber der Situation vor dem sandinistischen Triumph lassen sich in drei Punkten zusammenfassen. ${ }^{13}$

1. Die Guerillaverbände sind zielstrebiger, durchsetzungsfähiger, umsichtiger geworden. Eine Reihe von Fehlern, die Lamberg in seinem Resümee der Analyse der Guerillabewegungen der 60er Jahre für deren Scheitern verantwortlich macht, sind ausgeräumt, zumindest abgeschwächt worden (R. F. Lamberg 1971: 165 f.). In Anbetracht des militärisch-politischen Gewichts, das die Aufständischen in El Salvador und Guatemala erlangt haben, gehen etwa Vorwürfe wie der des individualistischen Volunta-

13 Die folgenden Punkte enthalten eine idealtypische Charakterisierung der Guerilla der späten 70er und frühen 80er Jahre. Teilweise sind die Unterschiede zu früheren Guerillabewegungen weniger ausgeprägt, als aufgrund der Darstellung angenommen werden könnte. Beispielsweise nahmen die Aufständischen in Venezuela und Argentinien, es wurde bereits darauf hingewiesen, nicht wenige der hier genannten Züge bereits vorweg. Die Ausführungen dieses Abschnitts stützen sich vor allem auf die einschlägigen Artikel und Berichte im "Latin America. Weekly Report" und der "Neuen Zürcher Zeitung" sowie auf von Klaus Lindenberg und Wolf Grabendorff freundlicherweise zur Verfügung gestelltes Quellenmaterial. Beiden sei an dieser Stelle für ihre Hilfe gedankt. 
rismus und ethischer Spontaneität, verfehlter taktischer Rezepte und eines blinden Vertrauens in die Eigendynamik der Gewalt heute ins Leere. Die Guerilleros haben sich in mehr als einer Hinsicht als sehr lernfähig erwiesen. Um nur einige der Züge zu nennen, in denen sich dieser Lernfortschritt manifestiert: Sie suchen nicht mehr in erster Linie die Unterstützung der in Lateinamerika chronisch schwachen Parteien, sondern arbeiten vor allem eng mit Massenorganisationen wie Gewerkschaften, Bauernverbänden, Angestelltenvereinigungen und kirchlichen Basisgruppen zusammen, wenden überhaupt der Mobilisierung der Unterschichten ungleich größere Aufmerksamkeit zu als ehedem; sie haben ihre frühere Theorielastigkeit abgebaut, konzentrieren sich vermehrt auf konkrete politische Ziele und sind besser dazu imstande, interne ideologische Differenzen im Interesse eines nach außen hin geschlossenen Vorgehens zu verdecken und zurückzustellen; sie operieren gleichzeitig auf mehreren Ebenen, einer politischen, einer kommunikativ-propagandistischen, einer militärischen, die jedoch aufeinander bezogen sind, ihren Gewaltaktionen liegt also ein strategisches Gesamtkonzept zugrunde; sie praktizieren intra- und international eine Politik breiter Allianzen, die auch gemäßigte Kräfte einschließt; sie versuchen die einseitige Abhängigkeit von transnationalen politischen Konstellationen und Stimmungen zu durchbrechen, indem sie durch gezielte Propaganda (in Form von eigenen Delegationen, Konferenzen, Informationsbroschüren u. dgl.) auf die internationale öffentliche Meinung Einfluß nehmen; und sie haben schließlich, was die Kampfstärke der Verbände, deren Versorgung, Organisation und waffenmäßige Ausstattung betrifft, jene Phase weit hinter sich gelassen, da Che Guevara mit einem kleinen Häufchen Getreuer etwas ziel- und orientierungslos durch den bolivianischen Urwald irrte. Mit einem Wort: Sie sind in mehreren Staaten zu einem Machtfaktor ersten Ranges aufgestiegen, während sie in der Vergangenheit überwiegend nur eine untergeordnete politische und militärische Bedeutung hatten.

2. Aufgrund ihres erhöhten militärisch-politischen Potentials sind die Guerillaverbände zu der Herausforderung für die den Status-quo verteidigenden Kräfte geworden, die Auseinandersetzung zwischen beiden Seiten hat eine in den 60er Jahren unbekannte Intensität erreicht. Schon im Rahmen der Stadtguerilla, Ende der 60er und in den frühen 70er Jahren (vor allem in Brasilien und Argentinien) eskalierten Gewalt und Gegengewalt zu neuen Dimensionen der Brutalität. Die terroristischen Anschläge der Stadtguerilleros, denen Staat und Gesellschaft viel hilfloser gegenüberstanden als den vergleichsweise harmlosen militärischen Angriffen von Landguerillaverbänden, reizten die Sicherheitskräfte zu noch schonungsloseren Vergeltungsschlägen. Heute sind eine extreme Rohheit, Grausamkeit und Rücksichtslosigkeit d. h. ein ausgeprägt menschenverachtender Zug zum allgemeinen Charakteristikum der bürgerkriegsähnlichen Kämpfe in Zentral- und Südamerika geworden. In diesem Punkt haben sich Stadt- und Landguerilla sehr einander angeglichen, ist nur ein geringfügiger Unterschied zwischen der Vorgehensweise der Rebellen und der Unterdrücker erkennbar (im allgemeinen setzen die Sicherheitskräfte und Todesschwadronen noch mehr als die Aufständischen auf die einschüchternde, zermürbende Wirkung des systematischen Terrors). Fairness, Ritter- 
lichkeit, Phantasie, die Achtung vor menschlicher Größe und der Respekt vor dem Leben Unbeteiligter, Elemente, die in den Auseinandersetzungen der 60er Jahre zumindest ansatzweise noch zu beobachten waren, sind verschwunden. Es gibt keine von beiden Seiten akzeptierten Spielregeln der Konfliktaustragung mehr, nur noch das Recht des in der jeweiligen Situation Stärkeren. Und es gibt auch keine am Kampf unbeteiligten Gruppen und Individuen mehr, da jedermann gezwungen wird, Stellung zu beziehen. Die gemäßigten Kräfte, welche sich diesem Polarisierungsdruck nicht beugen, sondern zwischen den Fronten zu vermitteln suchen, werden zermahlen. Das Volk, von beiden Seiten zugleich umworben und drangsaliert, sieht ihrem Wüten meist mit einer Mischung von Indifferenz und Schrecken zu und flieht, soweit möglich, um sich in Sicherheit zu bringen.

3. Diese Verschärfung des Konflikts läßt sich nicht allein aus der Verhärtung der Fronten innerhalb der vom Bürgerkrieg geschüttelten Länder erklären, sondern hängt daneben, insbes. in Zentralamerika, damit zusammen, daß die Auseinandersetzungen zunehmend eine transnationale Komponente erhalten. Dabei ist ein regionaler und ein globaler Aspekt zu unterscheiden. In regionaler Hinsicht ergeben sich immer dichtere Vermaschungseffekte zwischen den jeweiligen nationalen Exponenten des aufständischen Lagers einerseits, den Verteidigern des Status-quo andererseits. Die entsprechenden Gruppen jeder Seite unterstützen sich über die nationalen Grenzen hinweg und nehmen in den Nachbarländern gesammelte Erfahrungen rasch auf. Handelt es sich insoweit um eine intendierte gegenseitige Solidarisierung durch die am Kampf beteiligten Akteure, so haben diese auf den Stellenwert, der von den Supermächten dem Bürgerkrieg in Zentralamerika beigemessen wird, nur einen begrenzten Einfluß. Nachdem die Reagan-Administration entschlossen zu sein scheint, die Region zum Prüfstein des Anspruchs der USA auf Wiederherstellung ihrer internationalen Führungsposition zu machen, setzt sie alles daran, um einen Triumph der Revolutionäre in diesen Ländern zu verhindern. Die intensive wirtschaftliche Unterstützung und Waffenhilfe, die sie den Regierungen in diesen Ländern zukommen läßt, hebt den Konflikt auf eine neue, höhere Ebene und lädt ihn "künstlich" auf, verleiht ihm Züge eines Stellvertreterkrieges.

Wie wirken sich die beschriebenen Eskalierungsfaktoren auf die Chancen der Rebellen aus, werden diese dadurch erhöht oder vermindert? Eine allgemeine Antwort auf diese Frage geben zu wollen, ist ebenso riskant wie schwierig. Riskant, weil es sich um Entwicklungen handelt, die erst vor kurzem eingesetzt haben und deren Tragweite gegenwärtig kaum abzusehen ist. Die Schwierigkeit einer fundierten Lageeinschätzung resultiert u. a. aus der politisch-ideologischen Brisanz der Materie, die dem Bemühen um zuverlässige Informationen und ein ausgewogenes Urteil im Wege steht. Entscheidend dürfte sich das künftige Verhalten der USA auf die Erfolgschancen der beiden Seiten auswirken. Intensiviert Washington sein militärisches Engagement, indem es etwa eigene Truppen nach Zentralamerika entsendet, so dürfte es damit eine schwer kontrollierbare militärische Dynamik in Gang setzen (eine Einbeziehung von Honduras und Costa Rica in den Konflikt wäre fast unvermeidlich), an deren Ende nicht unbedingt 
ein Sieg der militärisch überlegenen Weltmacht stehen würde. Sollte die nordamerikanische Regierung dagegen auf eine direkte militärische Intervention verzichten, so ist schwerlich damit zu rechnen, daß die Rebellen in El Salvador und Guatemala in absehbarer Zeit einen definitiven Erfolg, verstanden als Eroberung der politischen Macht, für sich verbuchen können. Denn die meisten der eingangs dieses Abschnitts aufgezählten Lernprozesse und Verschiebungen betreffen lediglich operationale Aspekte, die weniger ins Gewicht fallen als strukturelle und akteurbezogene Faktoren. Stellt man primär auf die strukturellen Gegebenheiten sowie die soziopolitische Kräftekonstellation ab, so liegen weder in El Salvador noch in Guatemala, zumindest in kurzund mittelfristiger Perspektive, die Bedingungen für einen Sieg der Aufständischen vor.

\section{El Salvador}

In diesem Land haben die Bürgerkriegswirren in zwei Jahren (1980-82) rund 30000 Menschenleben gekostet. Die Zahl der Guerilleros beträgt rund 5000; während sie bis 1981 vorwiegend defensiv operierten, sind sie seit 1982 zu vermehrten offensiven Vorstößen übergegangen. Sie sollen mittlerweile rund ein Viertel des gesamten nationalen Territoriums unter ihrer Kontrolle haben. Sie haben Verbindung zu den Massenorganisationen (Gewerkschaften etc.), verfügen über einen eigenen Sender, sind in zahlreichen Staaten durch Delegationen vertreten, sind gut bewaffnet und organisiert und haben dank ihrer militärischen Effizienz den salvad. Streitkräften wiederholt empfindliche Verluste zugefügt, kurz sie entsprechen genau dem eben gezeichneten Bild einer gutgeschulten, straff geführten, politisch zielbewußten Kaderguerilla.

Blenden wir auf die im vorangegangenen Abschnitt herausgestellten Erfolgsdeterminanten der Guerilla zurück, so lassen sich schnell einige Faktoren nennen, die diesen Aufschwung und die gegenwärtige Machtposition der Aufständischen erklären helfen: der geringe Umfang, Entwicklungsrückstand und die intensive Außenabhängigkeit des Landes von den USA; die Existenz einer äußeren Anlehnungsmacht (neben Kuba), nämlich Nicaraguas, dessen Vorbildrolle - als Beispiel einer erfolgreichen Revolution fast noch mehr ins Gewicht fallen dürfte als seine Funktion als Schon-, Rückzugs- und Nachschubraum; eine gewisse diplomatische Unterstützung vonseiten der regionalen Mittelmächte (vor allem Mexikos) und bestimmter europäischer Regierungen (z. B. Frankreichs). Auch das Merkmal einer Legitimitätskrise liegt vor. Sie hängt mit dem Niedergang der staatstragenden vom Militär gestützten Partei, des PCN, seit Beginn der 70er Jahre, mit der sich verschärfenden Ungleichheit in den ländlichen Besitzverhältnissen, der zunehmenden Zwangsunterdrückung der Bevölkerung durch den staatlichen Sicherheitsapparat und der als Konsequenz des Bürgerkriegs sich beschleunigenden Wirtschaftskrise zusammen.

Ob man indes, wie im Falle des Somoza-Regimes von einem regelrechten Legitimitätsverfall des politischen Systems ausgehen kann, verbunden mit einem entsprechenden Legitimationszuwachs auf Seiten der Rebellen, ist fraglich. Die Tatsache, daß das 
Militär seit rund zehn Jahren nicht nur Statthalter der oligarchischen Gruppen, sondern eine relativ eigenständige politische Kraft ist, daß es sowohl innerhalb der Streitkräfte als auch im zivilen Lager stets minoritäre reformorientierte Strömungen gab, daß ab 1979 Reformen (z. B. eine Umverteilung des Landes) konkret in Angriff genommen wurden, daß im Frühjahr 1982 Wahlen stattfanden - all dies vermag die Legitimitätskrise zwar nicht zu heilen, verhindert jedoch, daß sie sich bis zu einem Punkt zuspitzt, wo die Mehrzahl der Bürger in einem bewaffneten Aufstand den einzig verbleibenden Weg zu einer Verbesserung der Verhältnisse sähe. Dieser Punkt ist auch deshalb noch nicht erreicht, weil die schwindende Legitimation der staatlichen Machtträger nicht von einem zunehmenden Vertrauen der Bevölkerung in die Ziele und Vorgehensweise der Aufständischen begleitet wird. Denn in der gewaltsamen Form der Durchsetzung unterscheiden sich beide Seiten nur wenig; je länger der Kampf andauert, je größer die Zahl der Opfer wird, desto mehr verbreitet sich vielmehr generelle Skepsis hinsichtlich des Sinns und Nutzens des blutigen Ringens.

Weil das bestehende Herrschaftssystem nicht endgültig diskreditiert ist, haben sich die gemäßigt-reformbereiten Kräfte (wobei vor allem an Teile der Mittelschicht und die Kirche zu denken ist) nur zu einem geringen Teil vom herrschenden Block losgesagt, um gemeinsame Sache mit den Rebellen zu machen. Im Gegensatz zur Situation ab 1974 in Nicaragua ist das Lager der bürgerlichen Gruppen in El Salvador nicht in nennenswertem Umfang gespalten. Ein gewisser Pluralismus, der auch in der Existenz mehrerer rechter Parteien seinen Niederschlag findet, zeigt nur Differenzen sekundärer Natur an, darf aber nicht als Ausdruck eines grundlegenden Dissenses mißverstanden werden. Mangels eines zumindest tendenziell sichtbaren sozio-politischen Ủbergewichts können die Insurgenten aus einer Verschärfung der Konfrontation keinen großen Vorteil ziehen; sie tragen durch eine solche Polarisierung unter Umständen dazu bei, daß die gemäßigten Gruppen zwischen den Fronten zerrieben werden, diese zu sich herüberzuziehen dürfte ihnen jedoch schwerlich gelingen. Was die Einstellung des breiten Volkes zu den Konfliktparteien angeht, so ist die Situation unübersichtlich und schwer einzuschätzen. Auf dem Lande scheinen die unterschiedliche Interessenlage der verschiedenen Unterschichtgruppen (Landarbeiter, Pächter, Kleinbesitzer) sowie der von rechtsextremen Terrororganisationen (ORDEN u. a.) ausgeübte Druck dafür gesorgt zu haben, daß eine Mobilisierung zugunsten der Guerilla auf breiterer Basis unterblieb; ${ }^{14}$ in den Städten verhinderten die Mitgliedschaft vieler Arbeiter in gemäßigten Gewerkschaften und deren Zersplitterung ein nennenswertes Engagement der Arbeiterschichten für die Sache der Revolutionäre. Insgesamt besteht nur eine geringe Wahrscheinlichkeit, daß es in absehbarer Zeit zu einer größeren Volkserhebung kommen könnte. Geängstigt durch die Gewaltausschreitungen verhält sich das Gros der Bevölkerung passiv oder versucht,

14 Rechtsterroristische Todesschwadronen sind für Guerillaverbände ein sehr ernst zu nehmender Gegner. Denn zum einen wirkt sich der von ihnen praktizierte Terror lähmend auf das politische Engagement der Bevölkerung aus, zum anderen sind sie nicht lokalisierbar und faßbar und deshalb ein schwierig zu bekämpfender Feind. 
durch Flucht innerhalb des Landes sowie über die Grenzen der Gefahr zu entgehen. Fehlen somit zumindest mittelfristig die Voraussetzungen für einen Erfolg der Aufständischen, so sind auf der anderen Seite auch die Streitkräfte außerstande, die Auseinandersetzung für sich zu entscheiden. Ihre numerische Úberlegenheit (rund $17000 \mathrm{Mann}$ ) reicht nicht hin, um gegen die Guerillatruppen einen vernichtenden Schlag zu führen und die "bef reiten Gebiete" zurückzuerobern, ganz abgesehen davon, daß es um ihre Kampfmoral und ihren Zusammenhalt schlecht bestellt ist. Die nordamerikanischen Beobachter, die zu dieser Feststellung gelangen müssen, wären jedoch schlecht beraten, wollten sie darauf hinwirken, daß US-Einheiten dem salvadorischen Militär zu Hilfe kommen. Denn auf diese Weise würde den Rebellen genau das zugespielt, was ihnen bislang abgeht: eine zugkräftige, eindeutige Definition des Gegners, gegen den sich der Befreiungskampf richten soll. Der von einer nordamerikanischen Intervention kurzfristig zu erwartende militärische Vorteil zugunsten der Sicherheitskräfte würde vermutlich die mittelfristig entstehenden Nachteile nicht aufwiegen: den zusätzlichen Legitimationsverlust der nationalen Regierung und der eigenen Streitkräfte, die sich als Marionetten der USA entpuppen würden und die vermehrte Chance der Mobilisierung breiterer Bevölkerungsgruppen unter antiimperialistischem Vorzeichen.

\section{Guatemala}

Der subversive Kleinkrieg nahm in diesem Land nicht erst im Gefolge der sandinistischen Revolution einen raschen Aufschwung, sondern hat eine eigenständige, in die frühen 60er Jahren zurückreichend Tradition. Damals bildeten sich in ländlichen Rückzugsgebieten mehrere Guerillaherde heraus, die sich teilweise längere Zeit behaupteten und nach F. R. Allemann (1974: 190) einer wirklichen Volks-Mobilisierung näher kamen als irgendeine andere Guerillabewegung Lateinamerikas. Nach ihrer Zerschlagung durch die Sicherheitskräfte ab 1967 kam der Widerstand gegen die ungerechte Besitz- und Herrschaftsordnung einige Jahre zum Erliegen, bis sich 1975 erneut eine bewaffnete Untergrundorganisation zu Wort meldete. Gegenwärtig existieren mehrere lose miteinander verbundene, vorwiegend jedoch unabhängig voneinander operierende Guerillaverbände, von denen der wichtigste der EGP ("Heer der Armen") ist. ${ }^{15}$ Nach ihrer Gesamtstärke noch etwas weniger zahlreich als die Aufständischen in El Salvador (rund 4000 Mann) haben sie es dennoch vermocht, ihre Aktivitäten auf fast sämtliche Provinzen des Landes auszudehnen. Der wichtigste Unterschied zur Situation in El Salvador besteht darin, daß es den guatemaltekischen Guerilleros noch nicht gelungen ist, "befreite Zonen" zu errichten, in denen die Herrschaftsgewalt dauerhaft in ihren Händen liegen würde. Die Frontlinien im Kampf zwischen den Rebellen und den Streitkräften haben sich noch nicht verfestigt, die Guerilla tritt nach klassischer Partisa- 
nenmanier primär durch Sabotageakte, plötzliche Hinterhalte, überraschende Ưberfälle und Anschläge, d. h. als Störfaktor in Erscheinung.

Die Geschichte der guat. Guerilla ist zugleich die Geschichte der Legitimitätskrise des guat. Staats. Etwas vereinfacht könnte man behaupten, das politische System habe sich nie von dem Verlust an Ansehen und Glaubwürdigkeit erholt, den es erlitt, als 1954 konservative Kräfte unter Zuhilfenahme der Unterstützung nordamerikanischer Truppen dem 10jährigen Reformexperiment (auch eine Landumverteilung war begonnen worden) der beiden sukzessiven Präsidenten J. Arévalo und J. Arbenz ein Ende bereiteten (E. Torres-Rivas 1980; J. Weaver: 1979). Die Guerilla der 60er Jahre war als unmittelbare Reaktion auf diesen Schock entstanden und auch die gegenwärtige Aufstandsbewegung geht indirekt auf ihn zurück. Die Antwort des Staates auf diese Herausforderungen bestand und besteht in erster Linie in nackter Gewaltanwendung. Psychische Einschüchterung und physische Eliminierung politisch Andersdenkender sind in Guatemala seit 15 Jahren zu einer so auffälligen Konstante des politischen Prozesses geworden, daß es bereits mehrere Aufsätze nordamerikanischer Sozialwissenschaftler gibt, die ausschließlich dieses Phänomen zum Gegenstand haben. (J. A. Booth 1980, D. L. Premo 1981). Offen bleibt dabei, inwieweit aus dem von Sicherheitskräften und Todesschwadronen gleichermaßen zu verantwortenden, durch einen fanatischen Antikommunismus zusätzlich gespeisten repressiven Terror auf strukturelle Stabilitätsprobleme des bestehenden Herrschaftssystems geschlossen werden kann. ${ }^{16}$ Der Anschein spricht eher gegen die Möglichkeit eines baldigen Zusammenbruchs der guat. Fassadendemokratie. Die guat. Streitkräfte gelten als schlagkräftiger und unabhängiger vom nordamerikanischen Beistand als das salvadoranische Militär. Auch die zivilen Machtgruppierungen, unter denen den Wirtschaftsverbänden ein besonders großer politischer Einfluß zukommt, wirken selbstbewußt und wenig angenagt von Legitimationszweifeln. Die Tatsache, daß die Spannungen, die sowohl im Militär als auch im zivilen Sektor als auch schließlich zwischen diesen beiden Blöcken auftreten, nicht verborgen, sondern in aller Offenheit ausgetragen werden, spricht gleichfalls gegen eine tiefergreifende Verunsicherung der herrschenden Kräftekoalition.

Die Guerilla der 60er Jahre fand den Zulauf, den sie in breiteren Bevölkerungsschichten hatte, vor allem bei Weißen und Mischlingen, während die rund $45 \%$ der Bevölkerung ausmachenden Indios in ihr nicht vertreten waren (Waldmann 1982: 110; N. Stoltz 1976). Die Situation hat sich insoweit grundlegend geändert, nach Schätzungen sollen heute viele Tausende von Indio-Familien als Unterstützungsbasis, teils auch als aktive Kombattanten, für den revolutionären Kampf gewonnen worden sein. In diesem Zusammenhang sei an die im vorangehenden Abschnitt vertretene These erinnert, noch nicht organisatorisch eingerahmte soziale Gruppen seien im allgemeinen aufgeschlossener für die Ziele der Guerilleros als bereits in Verbände bzw. Parteien eingegliederte Bevölkerungsteile - für die Indios trifft dies zweifellos zu, waren sie doch weder durch die

16 Dak von der politischen Führung ausgeübter Terror Ausdruck politischer Stabilität sein kann, also nicht unbedingt auf die Schwäche der Herrschenden schliel.sen lälıt, hat V. Walter 1969 nachgewiesen. 
Parteien noch die Gewerkschaften erfaßt. Bedenkt man weiter, daß die Indios in besonderem Maße von der Ausdehnung des Großgrundbesitzes auf Kosten kleinbäuerlicher und genossenschaftlicher Besitzformen betroffen waren, sie die Hauptleidtragenden der staatlichen und parapolizeilichen Willkür- und Sanktionsmaßnahmen sind sowie schließlich, daß in diesem Fall zu sozio-ökonomischer Ausbeutung und physischer Unterdrückung ein zusätzliches Moment rassischer Diskriminierung tritt, so erscheint es nicht ausgeschlossen, daß sie sich längerfristig, ungeachtet ihrer traditionell gemäßigten Haltung (Y. Le Bot 1979), in ein revolutionäres Potential verwandeln könnten. In diesem Fall würde sich das Kräfteverhältnis deutlich zugunsten der Aufständischen verschieben, die gegenwärtig den herrschenden Gruppen und dem Sicherheitsapparat noch klar unterlegen sind.

\section{Literatur}

Allemann, Fritz René: Macht und Ohnmacht der Guerilla, München 1974.

Anderson, Thomas A.: Politics in Central America, New York 1982.

Bernecker, Walter: Artikel "El Salvador", in: Waldmann, Peter (Hrsg.): Politisches Lexikon Lateinamerika, 2. Aufl., München 1982, S. 119-132.

Bieber, Horst: Nicaragua nach dem Sturz Somozas, Lateinamerika Berichte, 4. Jg., 25 (Sept. Okt. 1979), S. 16-25.

Booth, John A.: A Guatemalan Nightmare: Levels of Political violence 1966-1972, in: Journal of Interamerican Studies and World Affairs, Vol. 22, 1980, No. 2, S. 195-225.

Boris, Dieter: Nicaragua, in: Ders. u. Rausch, Renate (Hrsg.): Zentralamerika, Köln 1983, S. 249-345.

Boris, Dieter u. Rausch, Renate (Hrsg.): Zentralamerika, Köln 1983.

Bye, Vegard: The Success of the Nicaraguan Revolution: Why and How?, in: Ibero Americana. Nordic Journal of Latin American Studies, Vol. XI, 1-2, 1982, S. 3-16.

Castillo, Donald: Welche Faktoren haben den Erfolg der Revolution in Nicaragua möglich gemacht? Arbeitspapier für ein Expertengespräch der Friedrich-Ebert-Stiftung vom 9.-11. 3. 1981.

Delich, Francisco J.: Crisis y Protesta Social, Cordoba 1969-1973, 2. Aufl., Buenos Aires 1974.

Eckstein, Harry: On the Etiology of International Wars, in: Feierabend, Ivo u. a. (Hrsg.): Anger, Violence and Politics, New Jersey 1972, S. 9-30.

Ellner, Steve: Political Party Dynamics in Venezuela and the Outbreak of Guerrilla Warfare, in: Inter American Economic Affairs, Vol. XXXIV, 1980, No. 2, S. 3-24.

Evers, Tilman Tönnies: Militärregierung in Argentinien, Hamburg 1972.

Goldenberg, Boris: The Cuban Revolution and Latin America, 2. print. New York 1966.

Grabendorff. Wolf: Konstanten der Lateinamerikapolitik der USA, in: Lindenberg, Klaus (Hrsg.): Politik in Lateinamerika, Hannover S. 1971, S. 160-175.

Grabendorff. Wolf: Mittelamerika als internationale Krisenregion, in: Europa Archiv, Vol. 37, Nr. 8, April 1982, S. 247-258.

James, Daniel: The Peronist Left, 1955-1975, in: Journal of Latin American Studies, Vol. 8, 2 Nov. 1976, S. 273-296.

Jung, Harald: Nicaragua: Bereicherungsdiktatur und Volksaufstand, Frankfurt 1980.

Lamberg, Robert F.: Die castristische Guerilla in Lateinamerika, Vierteljahresberichte, Sonderheft 7, Hannover 1971.

Le Bot, Ivon: Paysannerie indienne et Mouvement social au Guatemala, in: Cahiérs des Amériques Latines, 1979, S. 185-195.

Leo Grande, William M.: The Revolution in Nicaragua: Another Cuba?, in: Foreign Affairs, Fall 1979, S. 28-50.

Lindenberg. Klaus: Zur Krise der revolutionären Linken in Lateinamerika: Das Beispiel Venezuela, in: Vierteljahresberichte, Nr. 33, Sept. 1968, S. 281-306. 
Mao Tse-Tung: Theorie des Guerilla-Krieges (mit einer Einleitung von Sebastian Haffner), Hamburg 1974.

Martín-Baró, Ignacio: La guerra civil en El Salvador, in: Estudios Centroamericanos, Jg. 36 (1981), Nr. 387/388, S. $17-32$.

Mc Donald, Ronald H.: El Salvador: The high cost of growth, in: Wiarda, J. Howard u. Kline Harvey F. (Hrsg.): Latin American Politics and Development, Dallas 1979, S. 389-398.

Millet, Richard: Guardians of the Dynasty: A History of the US created Guardia Nacional de Nicaragua and the Somoza Family, Maryknoll 1977.

Nohlen, Dieter u. Nuscheler, Franz: Handbuch der Dritten Welt, Bd. 3, 2. Aufl., Hamburg 1982.

Ortega, Humberto, Interview, durchgeführt von Marta Harnecker, Costa Rica 1980.

Payeres, Mario: Los Días de la Selva, Habana 1980.

Plant, Roger: Guatemala: Unnatural Disaster, London 1978.

Polo-Cheva, Demetrio u. Süßdorf, Erich: Nicaragua: die historischen Bedingungen einer demokratischen Revolution, in: Lateinamerika. Analysen und Berichte 4, Berlin 1980, S. 15-42.

Premo, Daniel L.: Political Assassination in Guatemala: A Case of Institutionalized Terror, in: Journal of Interamerican Studies and World Affairs, Vol. 23, No. 4 (1981), S. 429-458.

Rouquié, Alain: Pouvoir Militaire en Republique Argentine, Paris 1978.

Sieglin, Veronika: El Salvador, in: Boris, Dieter u. Rausch, Renate (Hrsg.): Zentralamerika, Köln 1983, S. 129-192.

Stoltz, Norma: La Minoría que es una Mayoría: Los Indios de Guatemala, in: Jonas, Susanne u. Tobis, David: Guatemala: Una Historia inmediata, Mexico 1976, S. 53-79.

Torres-Rivas, Edelberto: Vié et mort au Guatemala: Réflexions sur la crise et la violence politique, in: Amérique Latine (1980), S. 5-18.

Van Eeuwen, D.: Du Somozisme a u Sandinisme, in: Cultures et développement, Vol. XII, 3-4, 1980, S. $507-537$.

Waldmann, Peter: Strategien politischer Gewalt, Stuttgart 1977.

Waldmann, Peter: Ursachen der Guerilla in Argentinien, in: Jahrbuch für Geschichte von Staat, Wirtschaft und Gesellschaft Lateinamerikas, Bd. 15, 1978, S. 295-348.

Waldmann, Peter (Hrsg.): Politisches Lexikon Lateinamerika, 2. Auf1., München 1982.

Waldmann, Peter: Vergleichende Bemerkungen zu den Guerilla-Bewegungen in Argentinien, Guatemala, Nicaragua und Uruguay, in: Lindenberg, Klaus (Hrsg.): Lateinamerika. Herrschaft, Gewalt und internationale Abhängigkeit, Bonn 1982, S. 103-124.

Walter, E. Victor: Terror and Resistance. A Study of Political Violence with Case Studies of some Primitive African Communities, New York 1969.

Walker, Thomas, W.: Nicaragua: The Somoza family regime, in: Wiarda, Howard J. u. Kline, Harvey F. (Hrsg.): Latin American Politics and Development, Boston 1979, S. 316-331.

Weaver, Jerry L.: Guatemala: The politics of a frustrated revolution, in: Wiarda, J. Howard u. Kline, Harvey F. (Hrsg.): Latin American Politics and Development, Dallas u. a. 1979, S. 332-345. 
ment policies. Active popular participation is described as an indispensable element in basic-needs oriented rural development. Participation in turn requires political and administrative decentralization. Some comments on restrictions on decentralization in developing countries are given in conclusion of the theoretical considerations.

The last section discusses in some detail the strategic value of deconcentrating the administration to improve implementation capacity. Empirical evidence is drawn from the Zambian Decentralization Programme of January 1st, 1981.

\title{
Guerilla Movements in Latin America
}

\author{
By Peter Waldmann
}

The article discusses the conditions of growth and success of guerilla movements in Latin America. It is centred around three topical complexes. First, the principal causes of revolutionary upheaval in Nicaragua under the leadership of the Sandinists are described. In order to test the wider validity of the causal factors delineated with regard to Nicaragua, two further movements are analysed: Venezuela in the early 1960s, and Argentina in the first half of the 1970s. As a result of this comparison, five main conditions are identified for the success, of failure, of guerilla movements in Latin America. The final chapter examines the significance of the Sandinist victory in Nicaragua for the political and military situation in Central America; some conclusions are then offered on the prospects of the conflicts in El Salvador and Guatemala.

\section{Cuba's Legislative Decree No. 50/1982 - Castro's Genuflection Before Capitalism?}

\section{By Ludwig Gramlich}

Cuba enacted a legislative decree on foreign investment in early 1982. Its 50 sections may have been influenced by other joint venture legislation in socialist countries all over the world, but also by genuine Latin American conceptions. The Cuban decree contains rather detailed and relatively liberal provisions for foreign investors which, however, have to be completed by acts of public international law. Particularly in the field of taxation, unilateral fiscal incentives may sooner or later be maintained by tax treaties with home countries of foreign enterprises.

Another significant trait of the new Cuban foreign investment regulations are some provisions on labour law distinguishing between permanent contractual relations of Cuban employers and employees on the one side and temporary work of those employees under direction of foreign entrepreneurs. 